Prototyping real time engineering systems using Hatley \& Pirbhai's requirement model

Technical Report No 131

David A Fensome

April 1992 


\section{PROTOTYPING REAL TIME ENGINEERING SYSTEMS \\ USING HATLEY \& PIRBHAI'S REQUIREMENT MODEL}

\section{David A Fensome}

School of Information Sciences, Hatfield Polytechnic, Hatfield Herts AL10 9AB UK

Abstract A research programme is underway which is aimed at establishing ways of prototyping real time engineering systems using existing concurrent programming systems. The role of formal techniques in the development of prototypes, and its subsequent reuse in a well engineered product, is also an important theme. The first prototypes have used the Hatley \& Pirbhai Transformation Schema, and have been built using Ada. A case study is described.

The research shows that a prototype based on Hatley \& Pirbhai's requirement model would require a significant structural overhead, mainly because of the model 'execution' assumptions. Also Ada is an inappropriate language to use, mainly because the tasking overhead is too great, but also because the communications model differs from Hatley \& Pirbhai's model. Some future research direction are indicated.

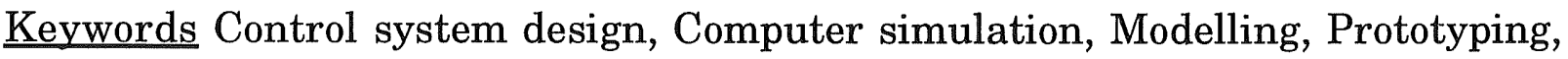
Real time computer systems, Software development, Software specification, Transformation Schema.

\section{INTRODUCTION}

A research programme is currently underway which is aimed at establishing ways of prototyping real time engineering systems. In particular

- To evaluate the suitability of existing concurrent programming 
systems

- To establish the role of formal techniques in the development of prototypes

- To identify ways that this formality could subsequently be reused in the development of a well engineered software product.

- To provide an environment to improve the prototyping process.

Prototyping in the context of this research means the examination of areas of uncertainty in the functional requirements by execution of a software model. Other aspects of prototyping such as target system performance and user interface considerations are regarded as separate problems, and are not considered. Real time engineering systems for this purpose covers that class of systems that are event driven, are (software) process oriented, and concerned with the control and monitoring of equipment in its widest interpretation (sometimes called 'reactive' systems). See (Harel, 1992) for a general discussion on modelling and prototyping of reactive systems.

The initial work is to build prototypes from requirements specifications expressed in natural language and diagrams. The first prototypes have used the Hatley \& Pirbhai Transformation Schema (Hatley, 1988), since this is based on well understood and widely used data flow techniques from Structured Systems Analysis (Yourdon, 1989), and have been implemented in Ada. The rationale for this strategy lies in the intuitive notion that mapping from a data flow model to a concurrent procedural language (Ada in this case) should be easy. Earlier work using Transformation Schema as a basis for prototypes can be found in (Coomber, 1990) where Smalltalk is used as a process definition language, and a Petri net execution approach has been used.

\section{THE CROOK PROOF VENDING MACHINE CASE STUDY}

The first prototype was built using the Hatley \& Pirbhai requirement model of the 'Crook Proof Vending Machine' (CPVM), and an Ada animation. The 
requirements statement and top level modelling is described in Chapter 28 of (Hatley, 1988), and briefly, is an attempt to build a vending machine that does not allow customers to fool the machine into giving product when it should not. The machine also has facilities for storing information on product quantity and price.

As the model was built assumptions were made about the mechanisms the system had to interface into, since these were not available in (Hatley, 1988), and were important in deriving the model. For example it was assumed each input coin fell into a coin hopper where physical measurements were made on the coin (producing the_object: PHYSICAL in Fig 1), and the coin was then routed to the appropriate coin register, or returned to the user as a 'slug'.

Extracting the data models using the requirement dictionary given in (Hatley, 1988) was hard, mainly because emphasis is given to flow (and data store) 'types' eg group/primitive, internal/external, control/data, discrete/continuous, whereas the 'attributes' name /units /range/resolution /rate contain most of the information on data types. In fact both objects and types appear as entries in the requirement dictionary, whereas separate entries in a types and a data dictionary proved much more helpful in building the prototype.

\section{USING THE REQUIREMENT MODEL AS A PROTOTYPE MODEL}

Intuitively it would seem that the two models should be the same and the animation could be coded directly from the requirement model. However there are some problems with this.

\section{Model Execution}

The requirement model is built with the intention of 'mental execution', with only fine grain execution (almost single step mode), and a lot of assumptions about the execution environment. Firstly, as a consequence of the infinitely fast execution of the processes in the requirement model, there is no interleaving of events and each event is processed atomically. The prototype model would have to guarantee 
this by serial feedback on completion of event processing, or by some overall parallel monitoring of completion of data flow. Also the process model is only feed forward, and any accidental feedback via a complex process network will not produce deadlock or instability (or the mental execution environment will 'sort it out'). Finally the activation/deactivation of sets of processes by the associated control model must be completed after the atomic processing of an event, and this therefore implies a separate control phase in the prototype model.

\section{Data Flow}

The requirement model data types require access mechanisms in the prototype model eg a flow of 'read_price' (see Fig 2) requires to be changed to a new type 'price X product' for the prototype model. Also continuous data flow requires special attention in the prototype model (see below), and data stores in general require mutual exclusion mechanisms for readers and writers.

The requirements dictionary with standard BNF like data dictionary notation was found to be satisfactory for a high level view of the system. However refinement of types and objects using the $\mathrm{Z}$ type system (Potter, 1991), and separate dictionaries for types and objects, allowed easy translation to Ada.

Finally data flow arrows entering a requirement model process do not necessarily correspond to Ada task entries. Sometimes an Ada task will be in the 'server' role (with entries), and sometimes active calling other tasks. A better graphical model based on Buhr's notation (Buhr, 1984) was found to be much more convenient for the prototype.

\section{USING ADA AS AN ANIMATION TOOL}

The requirement model uses hierarchical process decomposition which is cumbersome to emulate with Ada's tasking model. This is because each level in the requirement model requires an Ada task with a 'select' and multiple 'accepts' just to pass on data to lower levels in the hierarchy. 
package body context_data is

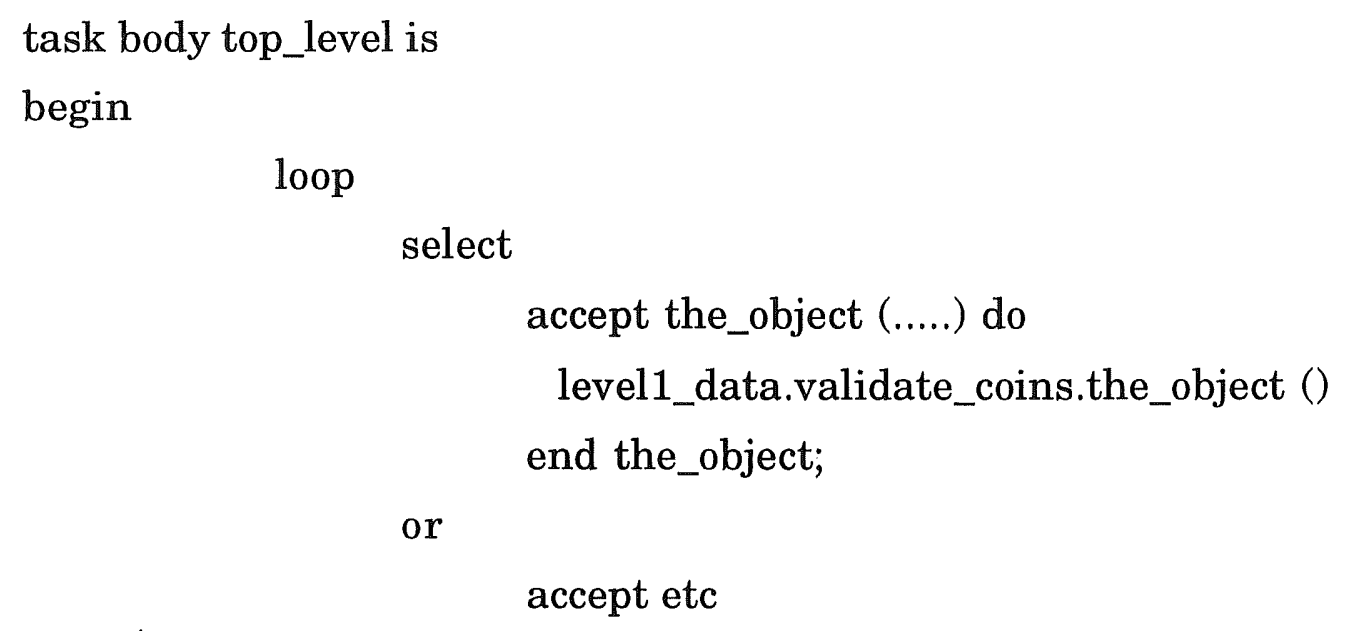

Fig. 3. An Ada implementation of a top level requirement process

\begin{abstract}
Also the possibility of deadlock with this structure is very high because of the additional rendezvous. This could be avoided by 'flattening' the process network, but this then means that large requirement models become potentially unmanageable. Also the positive advantage of automatic consistency checking between levels by the compiler ( the 'balancing' part of the requirement model build), would be lost.
\end{abstract}

This structural overhead, and associated deadlock risk, could be significantly reduced using OCCAM with named channels as parameters to lower level procedures (representing parallel processes), as shown in Fig. 4 below.

PROC level0 (CHAN OF INT coin, CHAN OF REAL32 price.....

PAR

$$
\begin{aligned}
& \text { level1.1 (coin, ....) } \\
& \text { level1.2 (price, ....) }
\end{aligned}
$$

PROC level1.1 (CHAN OF INT coin, .....) 
PAR

level1.1.1 (coin,

level1.1.2 (....

Fig. 4. Skeleton OCCAM process structure using channels

However the data typing facilities offered by OCCAM are significantly poorer than those of Ada.

Another problem using Ada is that the Ada task state is not the same as a requirement model process state. In the requirement model a process is activated and deactivated by the underlying control model (a finite state machine ), which effectively removes any communication with a deactivated process. In Ada a task runs forever, or until terminated in some way, and then completes. This means that entries have to be provided to activate and deactivate an Ada task, and in addition, to be able to check the state of a task using a complex select/loop structure for each requirement model process. A possible structure for each requirement model process is shown below. 


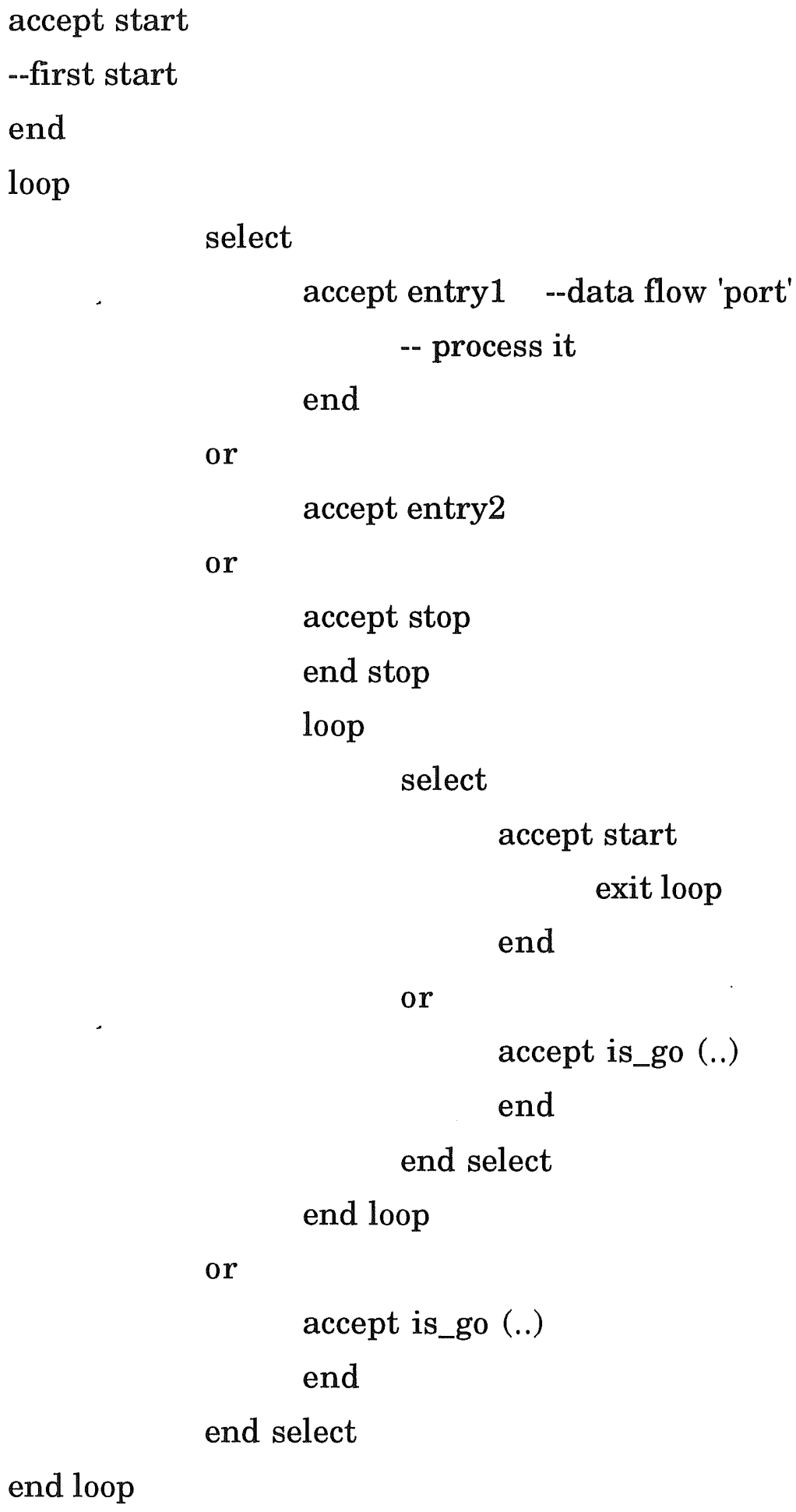

Fig. 4. Possible process state emulation with Ada. 
The Ada communication model is also different to the requirement model in several ways. Firstly communication is not via a named channel, but rather by a named port (the entry), giving the cumbersome Ada task structure mentioned above. Also Ada tasks must check whether a consumer task is active before communication is started, otherwise deadlock will occur. In addition it is desirable to include a single buffer in an Ada emulation, at least to decouple producer and consumer, but also to provide a simulation of a continuous data flow (ie to allow random re-reads of a data flow by a consumer process).

Finally there is no direct equivalent of the split or merge data flow operations that occur in the requirement model. These would have to be provided by yet another Ada task.

These communications differences indicate that a generic package is required for the Ada emulation, containing one task for each data flow, and generic parameters defining the data flow type and the entry name of the consuming task. This was built for unidirectional data flow, as shown in Fig. 5, but the Ada language would not allow a generic split or merge.

generic

type MESSAGE is private;

--data flow type

with procedure fire_entry (the_mess : in

MESSAGE);

--consumer entry name

with function is_go return BOOLEAN;

--process state check

package uni_df is

procedure put_df (fwd_mess : in MESSAGE);

function get_df return MESSAGE;

end uni_df; 
package body uni_df is

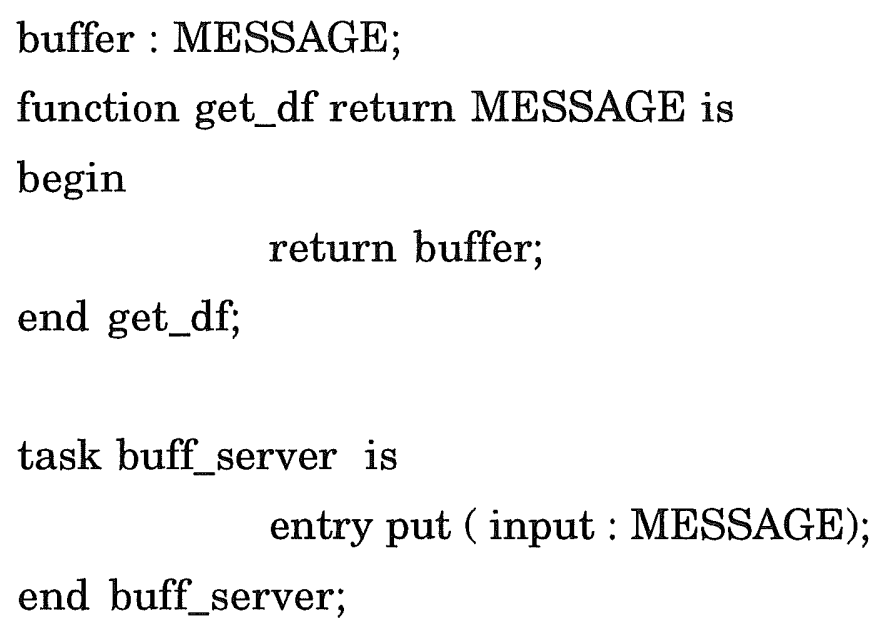

end uni_df;

Fig. 5. Ada Uni-directional data flow emulation 


\section{CONCLUSIONS}

Using Hatley and Pirbhai's requirement model as a basis for a prototype model is not quite so attractive as intuition might indicate, mainly because of the underlying 'execution' assumptions described above. In fact a prototype based on this model would require a significant structural overhead, almost like a conventional discrete event simulation package.

Using Ada to animate the prototype has shown that Ada is not an appropriate language, mainly because the tasking overhead is too great, and there is ample opportunity for deadlock. The tasking overhead occurs when implementing the requirement model process hierarchy, data flow and process state. One reason for this overhead is that the Ada communication model is many to one 'ported', whilst named channels are used in the requirement model. To some extent animations based on programming languages using channels, such as OCCAM, would be better, as long as the data modelling and generic facilities are reasonably good.

For these reasons it is not felt to be productive to pursue using Hatley and Pirbhai's requirement model as a prototype model, or its Ada animation.

Then next stage of the research will focus on a more appropriate (perhaps more abstract) requirement model without all the execution assumptions implicit in Hatley and Pirbhai's requirement model. It is felt that implementing prototypes from such models using conventional programming languages will be easier. Candidate requirement models are a $\mathrm{Z}$ model (Potter, 91) and a UNITY model (Chandy, 88), both based on mathematical models at a relatively high level of abstraction. However the issue of non determinism in these specifications, and how this is dealt with in the prototype, will have to carefully considered. The UNITY model has some advantage here, since the model is based on nondeterministic selection of statements executed in parallel and constrained by a fairness rule. . 


\section{REFERENCES}

Buhr, R. J. A. (1984) System Design with Ada Prentice Hall

Chandy and Misra (1988) Parallel Program Design Addison Wesley

Coomber, C. J. and Childs, R.E. (1990) A Graphical Tool for the prototyping of Real Time Systems ACM Sigsoft Vol15 No 2 Apr 1990

Harel, D. (1992) Biting the Silver Bullet IEEE Computer Jan 1992

Hatley, D.J. and Pirbhai, I. A. (1988) Strategies for Real-Time System Specification Wiley

Potter, B, Sinclair, J and Till, D (1991) An Introduction to Formal Specification and $Z$ Prentice Hall

Yourdon, E. (1989). Modern Structured Analysis Prentice Hall 


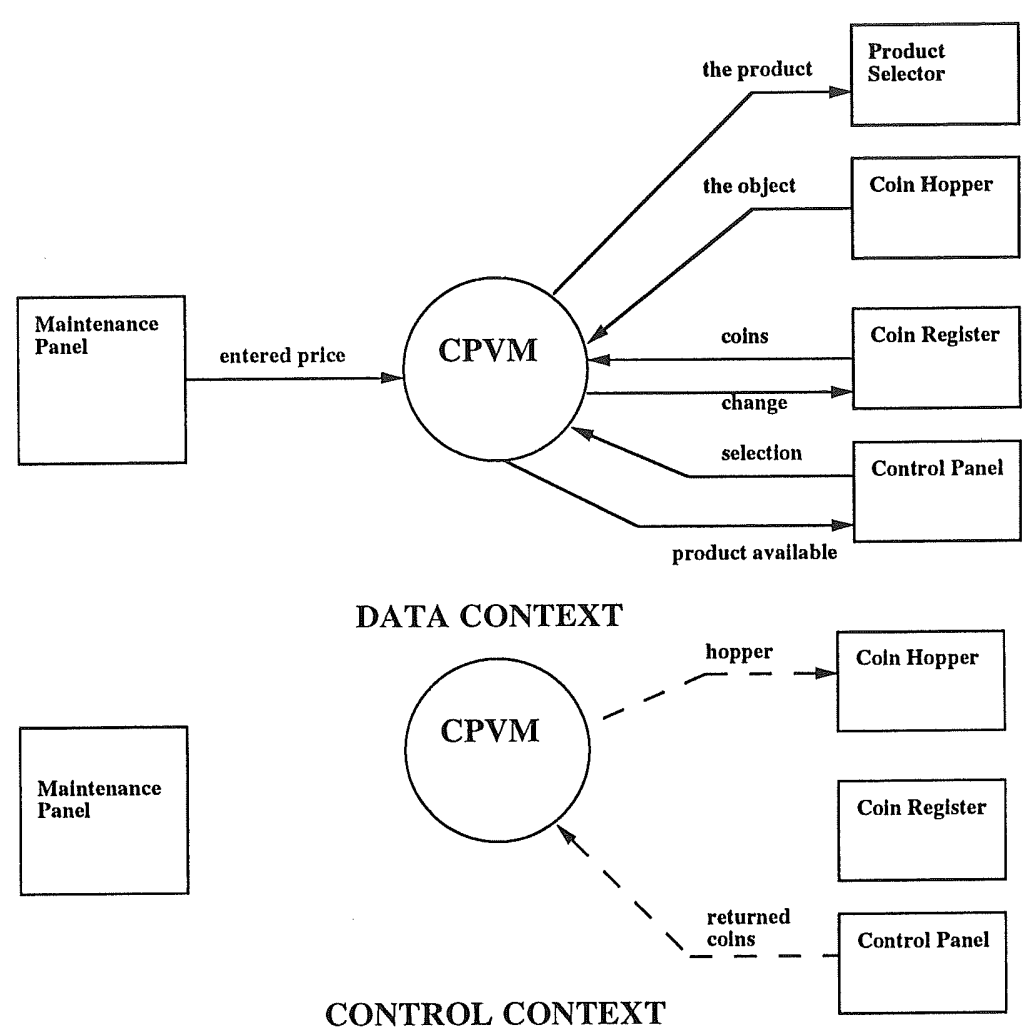

Fig. 1 Crook Proof Vending Machine

(after Hatley \& Pirbhai)

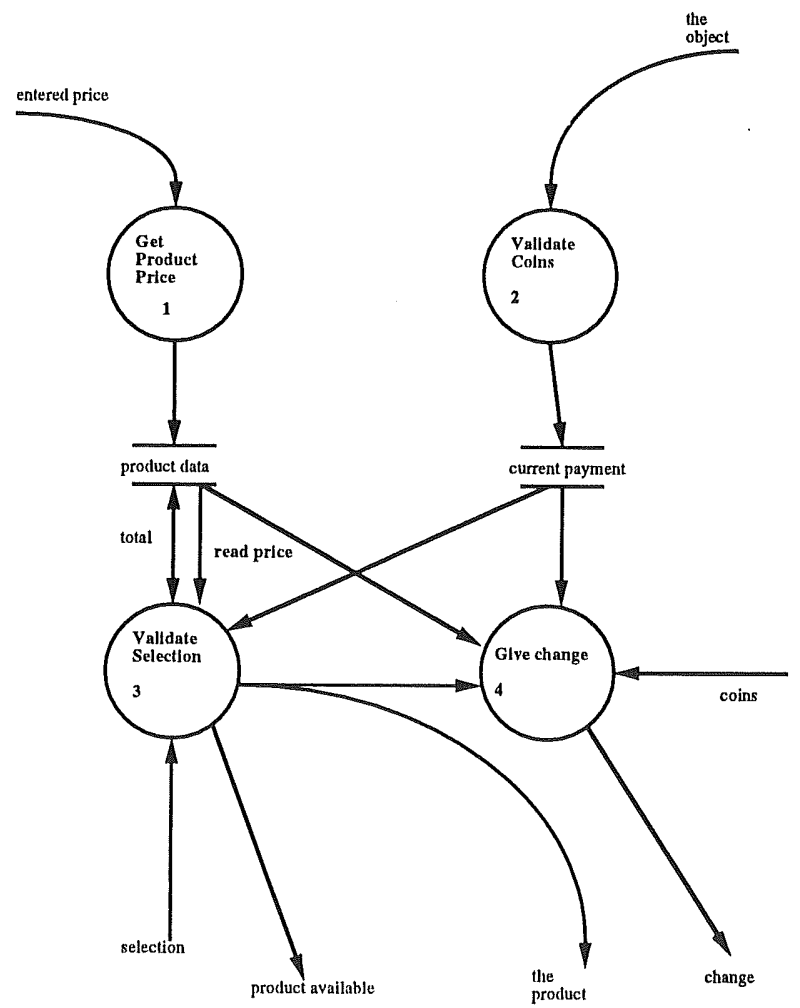

Fig. 2 CPVM DFD 0 Level 1 

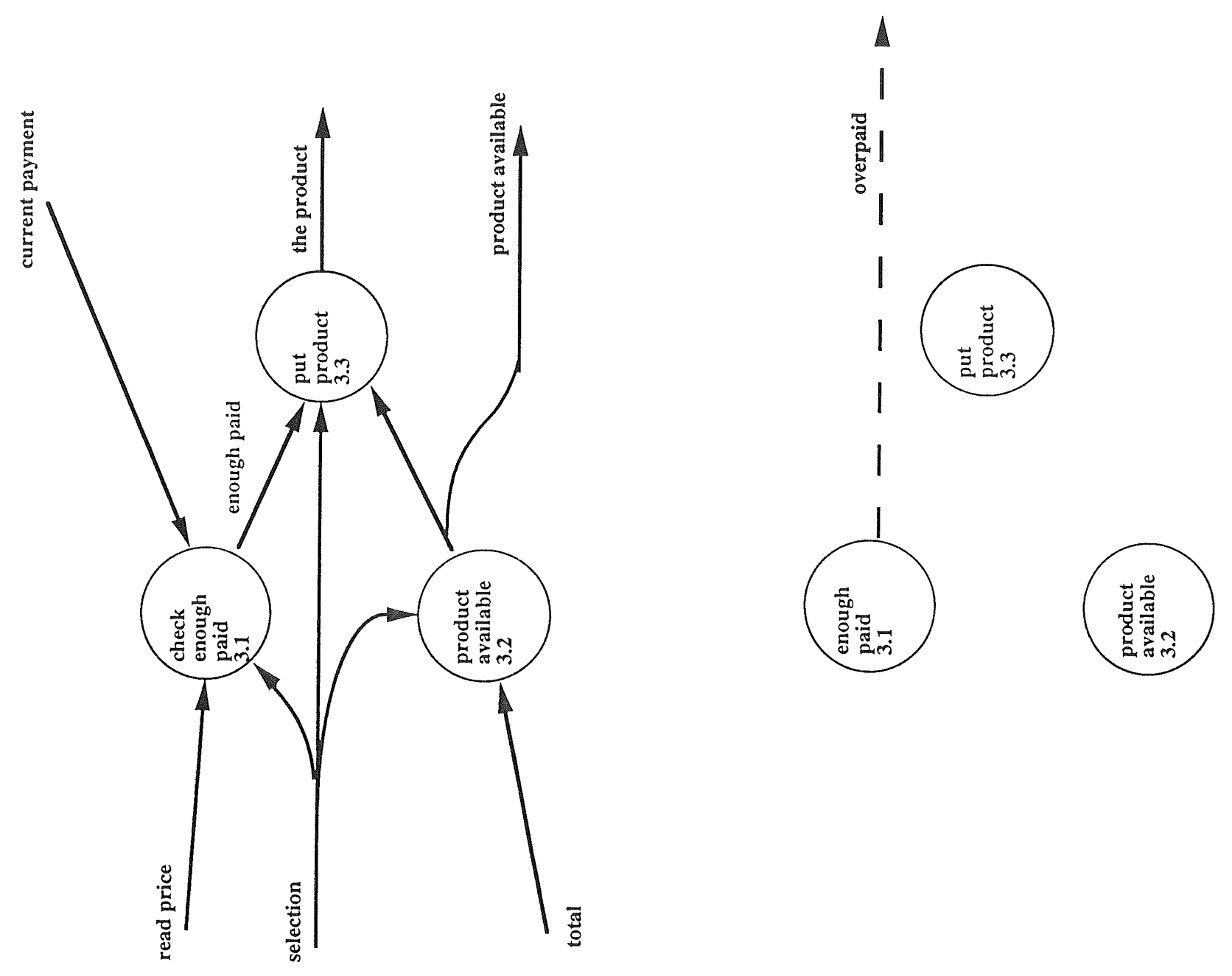

$\stackrel{\sqrt{0}}{\mathbb{0}}$

$\stackrel{0}{0}$
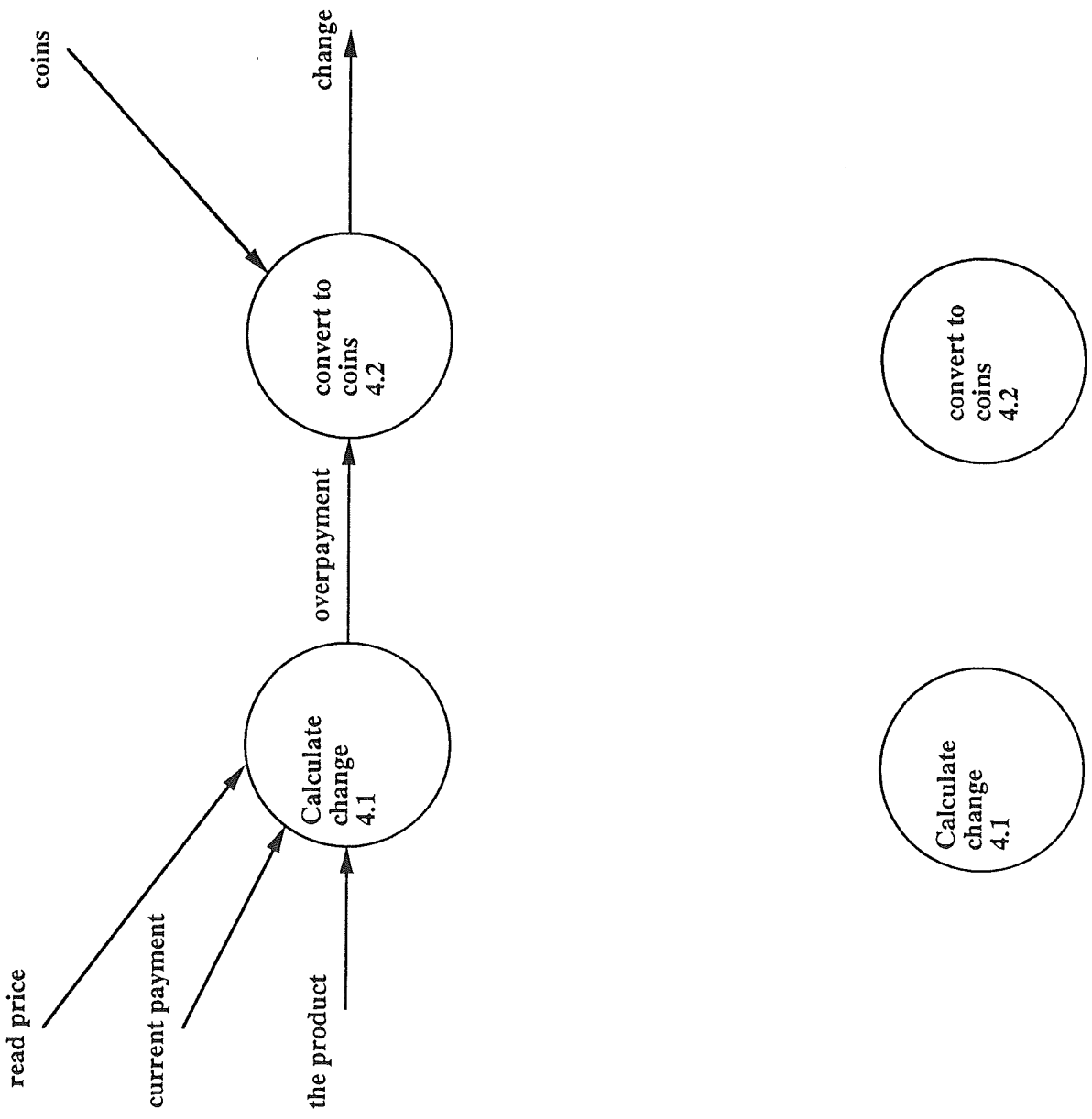

$\frac{0}{\mathbb{0}}$

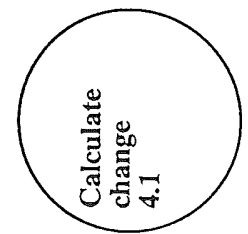

$\frac{7}{8}$ 


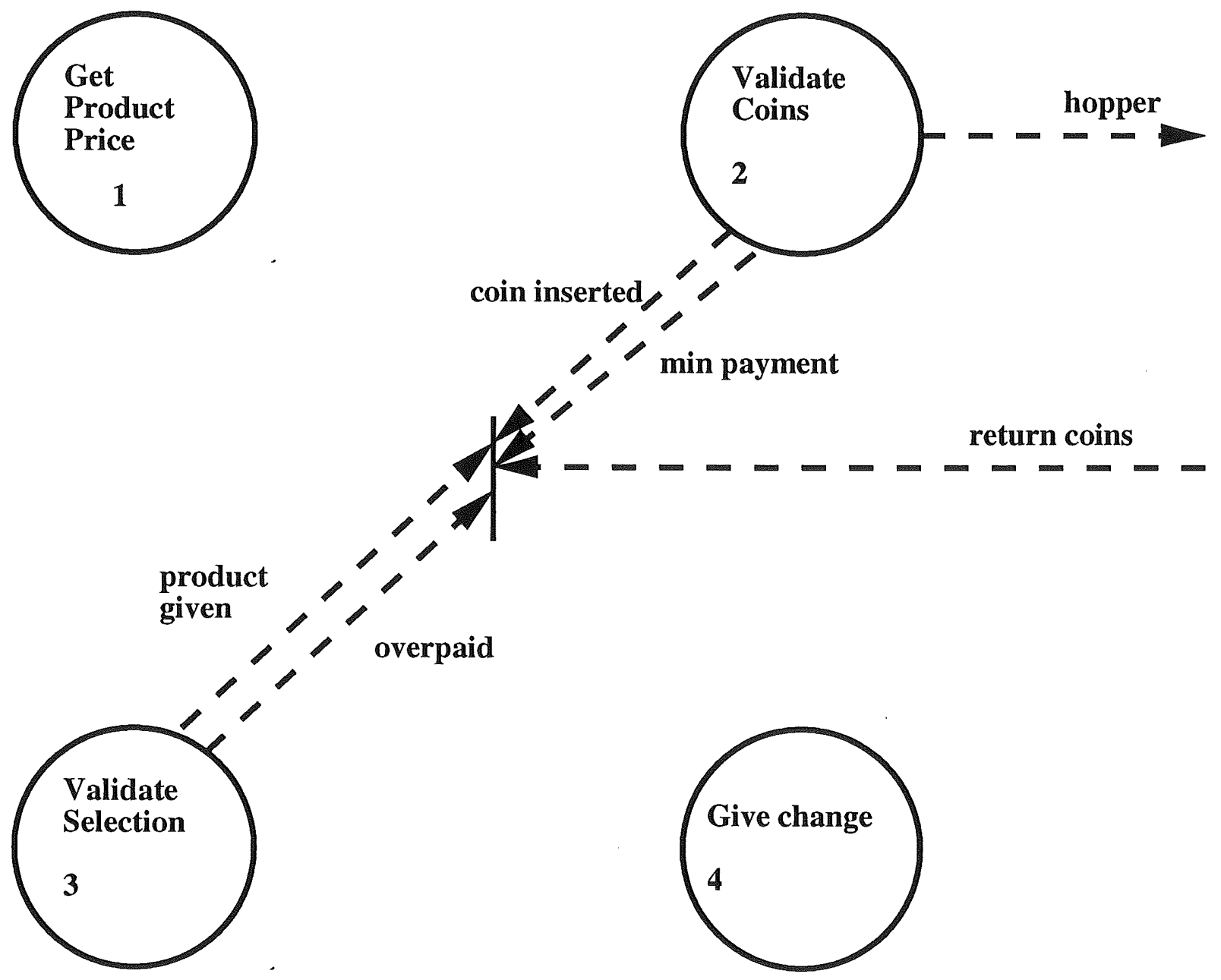




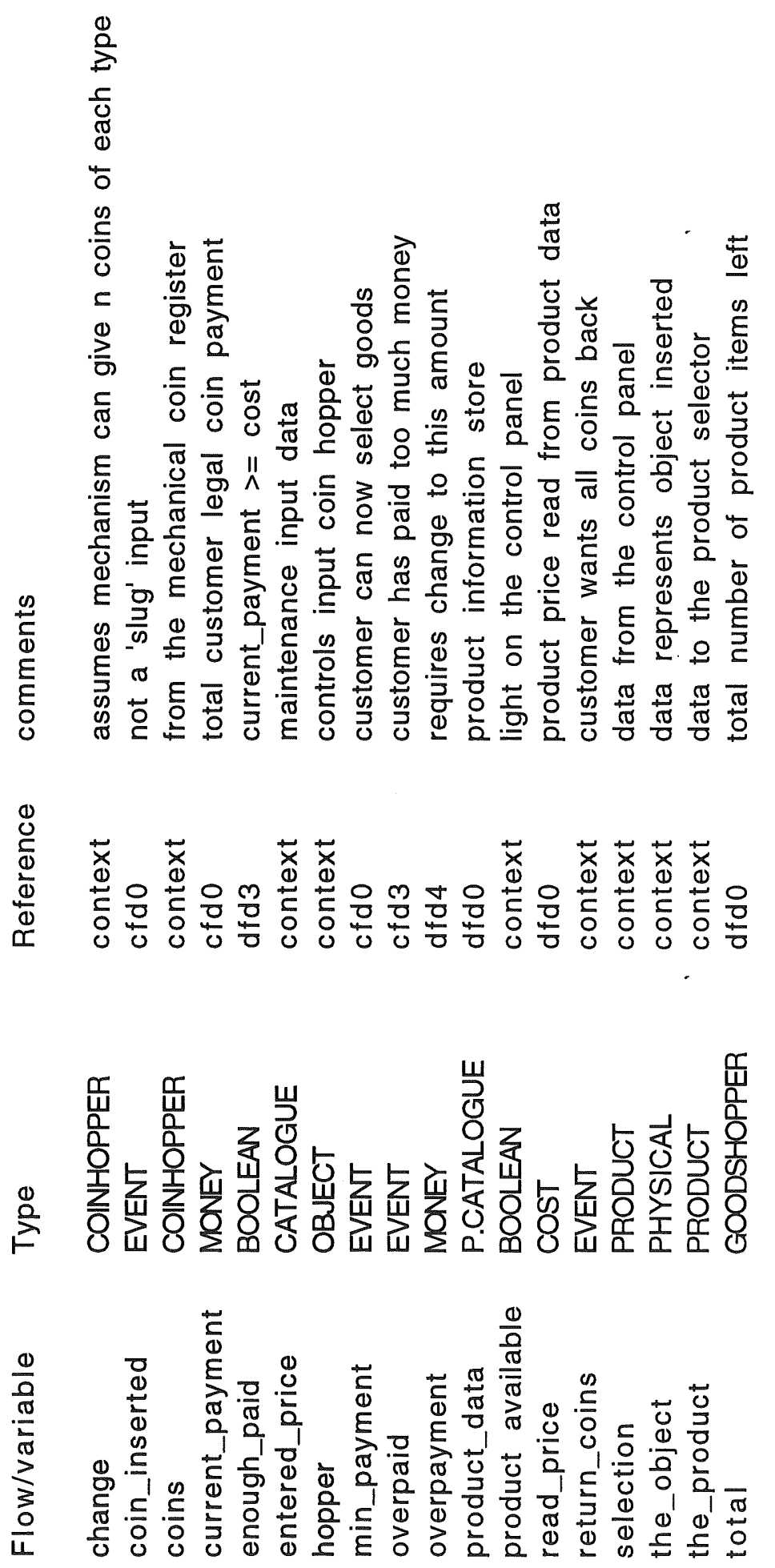


Type

CATALOGUE

COINCOUNT

COINHOPPER

COST

GOODSHOPPER

MONEY

OBJECT

PRODUCT

PHYSICAL

DIAMETER

WEIGHT

EVENT definition

comments

PRODUCT X COST X GOODSHOPPER

0 ..Maxcount

COINCOUNT $X$ COINCOUNT $X$ COINCOUNT 50ps $X 20 p s ~ X 10 p s$

Mincost..Maxcost

0 .. Maxhopper

Minmoney...Maxmoney

10pl20pl50plslug

Beer I Cola

DIAMETER $X$ WEIGHT

Mindia .. Maxdia

Minweight .. Maxweight

null assume just 2 products

no values 

